\title{
A summary of the National Institute for Health and Clinical Excellence (NICE) guidelines on lipid modification
}

\author{
Katherine Croom \\ CSF Medical Communications, Cheltenham, UK
}

Drugs in Context 2012

DOI: http://dx.doi.org/10.7573/dic.212226

Citation: Croom, K. A summary of the National Institute for Health and Clinical Excellence (NICE) guidelines on lipid modification. Drugs in Context: e212226. doi:10.7573/dic.212226

Copyright: this is an open access article published under the terms of the Creative Commons License Deed (CG BY-NC-ND 3.0) which allows you to share, copy, distribute and transmit the work provided it is properly attributed. You may not use this work for commercial purposes. For further information on commercial use, contact publisher@justmedicalmedia.com or go to wrwrw.drugsincontext.com/copyright.

NB: This article was originally published by CSF Medical Communications Ltd (CSF) in Drugs in Context 2008;4(2):177-84. Drugs in Context and all CSF copyrights were acquired by Just Medical Media Ltd in 2009. 


\title{
A summary of the National Institute for Health and Clinical Excellence (NICE) guidelines on lipid modification
}

\author{
Katherine Croom
}

CSF Medical Communications Ltd, UK

\section{PERSPECTIVE}

Cardiovascular disease (GVD), including coronary heart disease and stroke, is a major cause of morbidity and mortality in the UK, affecting as many as 5.6 million people in England alone. ${ }^{1}$ Despite reductions in mortality over the last 20 years, CVD remains the primary cause of death in the UK and is responsible for more than one-in-three deaths. A significant proportion of these deaths are in people aged less than 75 years; in 2005, CVD accounted for almost 57,000 premature deaths in the UK. ${ }^{1}$ The economic consequences of this level of morbidity are high; CVD cost the NHS around $£ 14.75$ billion in 2003, equivalent to around $£ 250$ per person. ${ }^{1}$

The extent of the medical and economic burden imposed by CVD, coupled with an increased understanding of risk factors and appropriate interventions, underlines the importance of preventative care in this field. Evidence-based practical guidance on how best to identify patients at risk, and what lifestyle advice and medical treatment to offer them, can facilitate the appropriate management of these patients. It is against this background that the NICE guideline Lipid modification: Cardiovascular risk assessment and the modification of blood lipids for the primary and secondary prevention of cardiovascular disease was published in May $2008 .^{2}$

\section{What does the new guideline add?}

Current health policy emphasises the need for high quality and appropriately targeted health care. For CVD prevention, measures must target not only patients with a history of CVD or diabetes but also those who are asymptomatic but at high risk of developing CVD. This dual emphasis on primary and secondary prevention was evident in the JBS 2 guideline on the prevention of $\mathrm{CVD}^{3}$ and also in the NICE guideline on the use of statins. ${ }^{4}$

While the JBS 2 guideline covered all key modifiable CVD risk factors, the current NICE guideline focuses specifically on the management of cholesterol. It presents guidance on the modification of lipids and sets out a strategy for identifying those people for whom primary prevention is appropriate.

\section{Role of primary care}

The important role of primary care in reducing GVD is acknowledged by the involvement of the Royal College of General Practitioners in the preparation of the guideline. GPs and practice nurses are well placed to be proactive in terms of CVD prevention. In addition to their role in the management of patients with established CVD, they are able to be involved in screening and providing risk assessment for asymptomatic people. 
This would be in line with the government initiative to introduce a nationwide cardiovascular screening programme for adults aged 40-74 years. ${ }^{5}$ Clearly, substantial additional resources would be necessary to provide a complete screening programme. However, targeted screening may be more easily achievable. The NICE guideline suggests that GPs use information contained in electronic patient records to identify patients who appear to be at highest risk, and target these patients for further assessment. This approach was found to be more cost-effective than screening by age or performing random screening. ${ }^{2}$

\section{THE NICE RECOMMENDATIONS}

The guideline provides recommendations for the identification and assessment of people at risk of CVD, and for a lipid modification strategy within the context of both primary and secondary prevention. It recommends treatment based on total cardiovascular risk rather than on isolated lipid levels. The lifestyle and drug treatment recommendations are in line with other guidelines, such as JBS 2 and the NICE guidance on the use of statins. ${ }^{3,4}$ This guideline does not cover people with diabetes or familial hypercholesterolaemia, who are dealt with in other NICE guidance (www.nice.org.uk).

\section{The guideline recommends treatment based on total cardiovascular risk rather than on isolated lipid levels.}

\section{Systematic identification of patients at risk}

One of the key features of the NICE guideline is the recommendation to use a systematic, rather than an opportunistic, approach to identify patients requiring primary prevention. The suggested strategy involves performing an initial estimate of risk using data contained in primary care records for patients aged 40-74 years, and then inviting those who appear to be at high risk (i.e. estimated 10 -year risk of $\mathrm{CVD}$ of $\geq 20 \%$ ) to undergo a formal risk assessment, with those with the highest score given priority. Patients with established CVD or known to be at high risk for other reasons, such as diabetes, would be excluded from this screening process. People aged $\geq 75$ would be considered to be at increased risk.

\section{Use a systematic, rather than an opportunistic, approach to identify patients requiring primary prevention.}

\section{Risk assessment}

The guideline recommends using 1991 Framingham 10-year risk equations for both the initial and the formal risk assessment, with some modifications applied during the formal assessment.

Of interest, the guidelines committee did consider the use of other risk equations; in particular those developed using UK populations, such as QRISK. However, in the end it recommended the well-established Framingham equation, with its known limitations, rather than a model that was still evolving. The committee recommended additional research should be done on the use of other equations.

The Framingham risk equation uses data on age, sex, systolic blood pressure, smoking, presence/absence of left ventricular hypertrophy, and total cholesterol and HDL cholesterol levels. If some information is not available during the initial risk assessment, values can be estimated using age- and sex-specific values drawn from population surveys, such as the Health Survey for England 2003. ${ }^{6}$ The guideline does not specify how to handle the absence of ECG results, but a default position of 'no LVH' would appear appropriate. ${ }^{7}$ During the formal risk assessment, evaluation of risk factors should include two systolic BP readings and the measurement of 
cholesterol levels, with nonfasting samples being adequate.

It is important to use clinical judgement in conjunction with the risk equation scores, particularly for patients with a score close to the 20\% threshold. Firstly, the Framingham 1991 risk equations may overestimate risk in contemporary UK populations, particularly in southern England. Secondly, in contrast, risk may be underestimated in some patients:

- People with a family history of premature CHD. The guideline recommends increasing the formal risk score by a factor of 1.5 for a history in one first-degree relative and by 1.5 to 2 if more than one first-degree relative is affected.

- Men of South Asian origin. The guideline recommends increasing the risk score by a factor of 1.4 during the formal assessment.

- Patients who have factors that predispose to GVD that are not covered by the risk equation, such as low socioeconomic status or severe obesity, or who are taking antihypertensive medications or have recently stopped smoking. NICE does not make specific recommendations for adjustments in these scenarios.

It is important to use clinical judgement in conjunction with the risk equation scores.

\section{Patient-centred care and communication}

The NICE guideline places emphasis on good communication with patients in order to arrive at a shared management plan, based on the premise that patients who are involved in decisions about their care are more likely to stick to a plan. It suggests discussing a patient's attitude to health and to risk as well as their reaction to any information they have received previously. Specific topics that need to be covered include:
Box 1. Communicating[risk[and[benefits]effectively

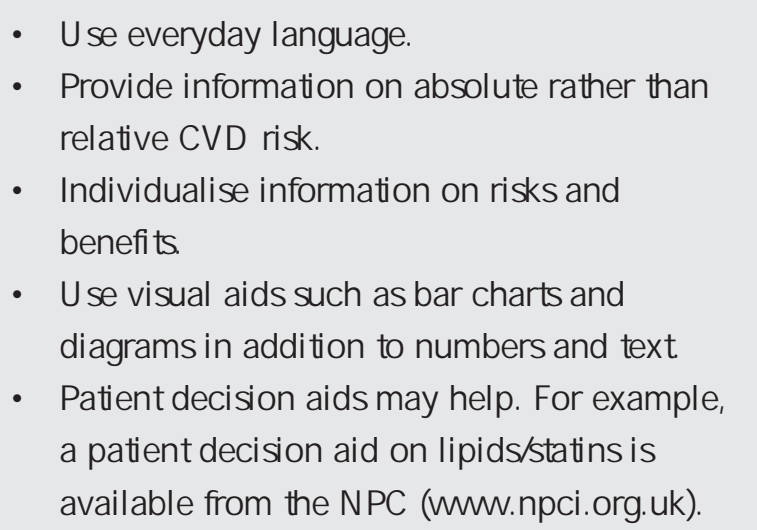

- Risk assessment - the concept of CVD risk, and what CVD risk assessment involves;

- Treatment - what lifestyle changes and drug treatment may be necessary;

- Initiating or changing drug therapy - the risks and benefits of treatment.

The guideline provides advice on how to communicate this information clearly (see Box 1).

The guideline emphasises that patient preference should be considered, and patients should be given the option of declining formal risk assessment and treatment. A note of the discussions held and the patient's decision should be made in the patient records. In the event of refusal, patients should be advised to consider the matter again in the future, and it would presumably be sensible to flag the patient's record for follow up.

The guideline emphasises that patient preference should be considered.

\section{Lifestyle changes}

Lifestyle advice should be provided to all patients at high risk or with existing CVD. The recommendations in the NICE guideline are in line with those provided in JBS 2, with the exception that NICE does not mention dietary 
salt, whereas JBS 2 recommends a limit of $<6 \mathrm{~g}$ sodium chloride per day. ${ }^{3}$

Key recommendations include:

- Eat a cardioprotective diet that is low in saturated fat and cholesterol, includes $\geq 5$ portions of fruit and vegetables each day and $\geq 2$ portions of oily fish each week.

- Limit alcohol to a maximum of 3-4 units/day for men or 2-3 units/day for women.

- Stop smoking.

- Undertake 30 minutes of moderate intensity exercise on $\geq 5$ days each week.

Patients can be referred to government websites for detailed information about diet (www.eatwell.gov.uk/healthydiet; www.5aday. nhs.uk). Additional advice on managing smoking cessation, obesity and physical activity is available from NICE (www.nice.org.uk).

\section{Lipid modification therapy}

Drug recommendations in the NICE guideline are based on evidence of clinical benefit on CVD morbidity and mortality and lowest acquisition cost and are in line with the advice provided in the NICE technology appraisal on statins. ${ }^{4}$

Prior to commencing drug treatment, the guideline advises that secondary causes of dyslipidaemia should be excluded, and fasting lipid levels should be measured (to provide a baseline against which to measure progress). The management of other modifiable risk factors and comorbidities should be optimised before starting drug therapy for primary prevention; however, treatment for secondary prevention should not be delayed by this.

Statins are the therapy of choice for both primary and secondary prevention of CVD, although there are some differences in the overall recommendations for these groups of patients. In both scenarios, treatment should commence with simvastatin $40 \mathrm{mg}$. In the event of contraindications or potential drug interactions, a lower dose or an alternative can be prescribed. Ezetimibe can be considered for patients with primary hypercholesterolaemia who have contraindications to, or are intolerant of statins. This is in line with NICE guidance on the use of ezetimibe. ${ }^{8}$

Key points relating to therapy for primary prevention include:

- People with a 10 year risk of $\geq 20 \%$ should be treated with simvastatin $40 \mathrm{mg}$.

- The dose of simvastatin should generally not exceed $40 \mathrm{mg}$ and other high-intensity statins (i.e. any statin with a greater cholesterollowering effect than simvastatin $40 \mathrm{mg}$ ) should not generally be used.

- Target levels for total and LDL cholesterol are not set, because there are no suitable data on the relationship between particular reductions in cholesterol level and clinical events from primary prevention clinical trials. However the aim of treatment is to reduce total and LDL cholesterol levels, reduce triglyceride levels and increase HDL cholesterol levels.

- Follow-up measurement of lipid levels is not considered necessary, and any review should be agreed between the patient and practitioner. In other words this guidance appears to make the assumption that treatment with simvastatin $40 \mathrm{mg}$ will be effective enough unless adverse events or other clinically relevant changes occur.

Key points relating to therapy for secondary prevention include:

- Patients with clinical evidence of CVD should be treated with simvastatin $40 \mathrm{mg}$, except those with an acute coronary syndrome who should receive a high-intensity statin.

- Targets of total cholesterol $<4 \mathrm{mmol} / \mathrm{L}$ or LDL cholesterol $<2 \mathrm{mmol} / \mathrm{L}$ are recommended, which are in agreement with JBS $2{ }^{3}$ 
- If target levels are not achieved, treatment with simvastatin $80 \mathrm{mg}$ (or a drug of similar efficacy and acquisition cost) can be considered in light of a patient's informed preference, comorbidities, other drug therapy and benefits and risks.

- More than half of patients with stable coronary artery disease will not achieve these target levels even at the higher dose. Consequently, the guideline recommends using an audit level of total cholesterol $5 \mathrm{mmol} / \mathrm{L}$.

Use of other lipid-lowering drugs:

- In the NICE technology appraisal on ezetimibe in primary hypercholesterolaemia published in November 2007, NICE recommends ezetimibe as monotherapy in the event of contraindications or intolerance to statins. ${ }^{8}$ It also recommends ezetimibe in combination with initial statin therapy in patients whose serum total cholesterol or LDL-C concentration is not appropriately controlled after dose titration or where titration is limited by intolerance to the initial statin therapy and consideration is being given to switching to an alternative statin. ${ }^{8}$

- Fibrates and anion exchange resins should only be considered if statins are not tolerated, in which case they can be used for primary or secondary prevention.

- Nicotinic acid can be considered for secondary prevention if statins are not tolerated, but is not recommended for primary prevention.

- Combination therapy with a statin plus a fibrate, anion exchange resin or nicotinic acid is not recommended for primary prevention.

\section{PRACTICAL IMPLICATIONS}

There remains considerable room for improvement in cardiovascular health in the UK. Widespread implementation of the preventative measures recommended in the NICE guideline would go a long way towards achieving this.
However, there are several issues to consider.

The recommendation to perform systematic screening to identify candidates for primary CVD prevention carries heavy resource implications in terms of time, manpower and costs for the NHS and primary care in particular. It is estimated that adopting the 20\% 10-year risk as the threshold for intervention could lead to 4.7 million asymptomatic people being considered for treatment for primary prevention, based on use of the Framingham equation. ${ }^{9}$ However, this number could be reduced if patients are provided with sufficient information and support to enable them to make healthier lifestyle changes.

Convincing people who feel well of the need for lifestyle changes or drug treatment is not always easy, and the guideline emphasises the importance of informed discussion, patients' informed preference and shared management plans. While many patients will base their decision on what they are told by healthcare staff during consultations, some patients are also likely to obtain information from other sources, such as newspapers, magazines and the internet. The guideline acknowledges that patients may have their own ideas and recommends that a patient's 'informed preference' is taken into account when negotiating and agreeing a management plan. Whilst this may raise some concerns about the amount of time it could take to discuss the finer points of treatment options with patients, it is generally accepted that well informed patients with whom a shared management plan has been negotiated are more likely to comply with the action points agreed.

\section{A patient's 'informed preference' should be taken into account when negotiating and agreeing a management plan.}

With respect to covering the costs associated with implementing the guidelines in primary care, QOF indicators exist for secondary prevention of coronary heart disease and for stroke/transient 
ischaemic attacks, including several relating to lipid levels. Currently, primary CVD prevention is not covered by QOF, although it has been proposed that indicators should be introduced. ${ }^{10}$ If this goes ahead, payments for achieving quality points should help offset the additional resource burden and potentially increase practice revenues. Clearly, the time and resources needed to implement the guideline recommendations will need to be considered carefully by practices. A costing template is available on the NICE website, which can be used to estimate the costs at a local level (www.nice.org.uk/CG67).

Currently, the only advice on audit criteria provided in the guideline is to use an audit level of total cholesterol $5 \mathrm{mmol} / \mathrm{L}$ for the group of patients being treated for secondary prevention. This is in line with current QOF indicators.

With regard to the practicalities of following the NICE guidelines, algorithms summarising the primary and secondary prevention pathways are provided in the guideline. Risk calculation charts are not included; however, many practice computer systems have software that can help calculate 10-year CVD risk. ${ }^{11}$ JBS 2 CVD risk prediction charts, which are based on the 1991 Framingham equations, are available in the BNF or on the British Hypertension Society (BHS) website (www.bhsoc.org), although they do not include LVH. An online risk calculator that includes all of the parameters proposed in the NICE guideline is also available on the BHS website.

The outcome data we now have on the clinical benefits of reducing LDL cholesterol and triglycerides and raising HDL cholesterol levels is compelling. ${ }^{2}$ Consequently, adherence to the recommendations in the NICE guideline on lipid modification should lead to long-term benefits in terms of improved cardiovascular health and reduced mortality. The guidance provided will be particularly useful to primary care professionals, as they play a key role in preventative cardiovascular care.

\section{KEY POINTS}

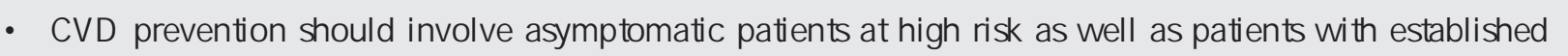
CVD.

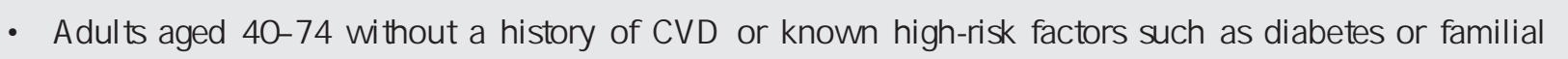
hypercholesterolaemia[\$hould[be[considered[for[primary[CVD[prevention.

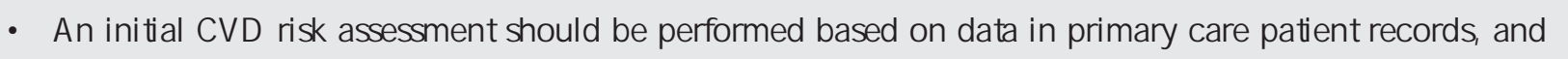

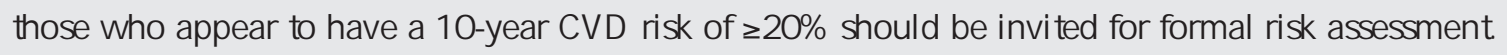

- $\square$ D Decisions $\square$ about $\square$ formal $\square$ risk $\square$ assessment $\square$ and $\square$ treatment $\square$ shoul $\square \square$ be $\square$ based $\square$ on $\square$ shared $\square$ decision-making $\square$ between[patients[and[healthcare[professionals.

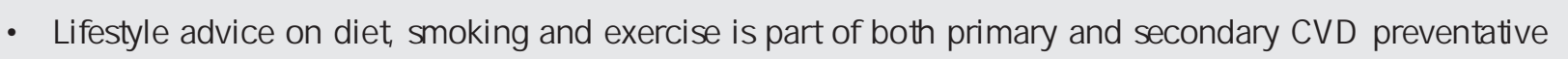
care.

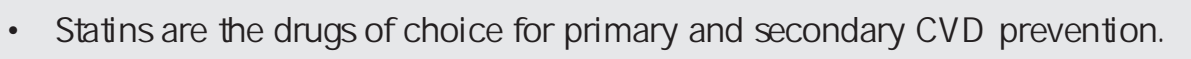

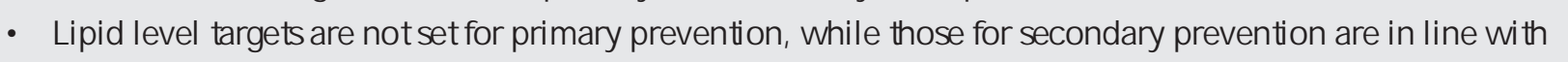
JBSRRguidelines. 


\section{GUIDELINES}

\section{REFERENCES}

1 British Heart Foundation. 2008 Cardiovascular disease statistics. Available from: www.bhf.org.uk.

2 National Institute for Health and Clinical Excellence (NICE). NICE clinical guidance 67. Lipid modification: cardiovascular risk assessment and the modification of blood lipids for the primary and secondary prevention of cardiovascular disease. Available from: www.nice.org.uk.

3 Joint British Societies' guidelines on prevention of cardiovascular disease in clinical practice. Heart 2005; 91 (Suppl 5): v1-52.

4 National Institute for Health and Clinical Excellence (NICE). NICE technology appraisal 94. Statins for the prevention of cardiovascular events. Available from: www.nice.org.uk.

5 Department of Health. Putting prevention first. Vascular checks: risk assessment and management. 1 Apr 2008. Available from: www.dh.gov.uk/publications.

6 National Centre for Social Research. Health Survey for England 2003. Department of Health 2004. Available from: www.dh.gov.uk/

7 Marshall T. The use of cardiovascular risk factor information in practice databases: making the best of patient data. $\mathrm{Br} \mathcal{J}$ General Practice 2006; 56 (529): 600-605.
8 National Institute for Health and Clinical Excellence (NICE). NICE technology appraisal 132. Ezetimibe for the treatment of primary (heterozygous-familial and nonfamilial) hypercholesterolaemia. Available from: www.nice. org.uk.

9 Hippisley-Cox J, Coupland C, Vinogradova Y et al. Derivation and validation of QRISK, a new cardiovascular disease risk score for the United Kingdom: prospective open cohort study. BMF 2007; 335 (7611): 136. Epub 2007 Jul 5.

10 National Primary Care Research and Development Centre. Cardiovascular disease primary prevention: quality and outcomes framework. Oct 2007. Available from: www. npcrdc.ac.uk.

11 Chauhan U. Cardiovascular disease prevention in primary care. Br Med Bull 2007; 81: 65-79

\section{ABBREVIATIONS}

BNF, British National Formulary; CVD, cardiovascular disease; HDL, high-density lipoprotein; JBS, Joint British Societies; LDL, low-density lipoprotein; LVH, left ventricular hypertrophy; NICE, National Institute for Health and Clinical Excellence; NPG, National Prescribing Centre; QOF, Quality and Outcomes Framework. 